\title{
Durable Complete Response With Immune Checkpoint Inhibitor in Breast Cancer With High Tumor Mutational Burden and APOBEC Signature
}

Saranya Chumsri, MD; Ethan S. Sokol, $\mathrm{PhD}^{2}$; Aixa E. Soyano-Muller, $\mathrm{MD}^{1}$; Ricardo D. Parrondo, $\mathrm{MD}^{1}$; Gina A. Reynolds, APRN ${ }^{1}$; Aziza Nassar, $\mathrm{MD}^{3}$; and E. Aubrey Thompson, $\mathrm{PhD}^{4}$

\section{ABSTRACT}

Increasing data support the importance of preexisting host immune response and neoantigen burden for determining response to immune checkpoint inhibitors (ICls). In lung cancer and melanoma, tumor mutational burden (TMB) has emerged as an independent biomarker for $\mathrm{ICl}$ response. However, the significance of TMB in breast cancer, particularly in the context of PD-L1 negativity, remains unclear. This report describes a patient with HER2-negative breast cancer with high TMB and an apolipoprotein B mRNA editing enzyme, catalytic polypeptide-like (APOBEC) trinucleotide signature; her disease was refractory to multiple lines of treatments but achieved durable complete response using $\mathrm{ICls}$ and capecitabine. Additional analysis of the tumor revealed a low amount of stromal tumor-infiltrating lymphocytes (sTILs) and PD-L1 negativity, reflecting a poor preexisting host immune response. In collaboration with Foundation Medicine, comprehensive genomic profiling from 14,867 patients with breast cancer with the FoundationOne test was evaluated. Using the cutoff of $\geq 10$ mutations/megabase (mut/Mb) for high TMB, PD-L1 positivity and TMB-high populations were not significantly overlapping (odds ratio, 1.02; $P=.87$ ). Up to $79 \%$ of TMB-high tumors with $>20$ mut/Mb were PD-L1-negative. Our study highlights that despite having low TILs and PD-L1 negativity, some patients may still experience response to $\mathrm{ICls}$.

J Natl Compr Canc Netw 2020;18(5):517-521 doi: $10.6004 /$ jnccn.2020.7543

\footnotetext{
${ }^{1}$ Robert and Monica Jacoby Center for Breast Health, Mayo Clinic, Jacksonville, Florida; ${ }^{2}$ Foundation Medicine, Cambridge, Massachusetts; and ${ }^{3}$ Pathology Department, and ${ }^{4}$ Department of Cancer Biology, Mayo Clinic, Jacksonville, Florida.
}

\section{Case Presentation}

A white woman aged 66 years initially presented with a large fungating right breast mass with bleeding requiring blood transfusion. CT scan of the chest, abdomen, and pelvis showed a $14-\mathrm{cm}$ mass in her right breast along with bulky right axillary lymphadenopathy. A punch biopsy of the tumor revealed grade 3 infiltrating ductal carcinoma, estrogen receptor (ER)-positive by immunohistochemistry (IHC) at $76 \%$ to $100 \%$, progesterone receptor (PR) focally positive at $10 \%$, HER2 nonamplification by fluorescence in situ hybridization, and a Ki-67 level of $70 \%$. Further staging using a PET/CT scan, bone scan, and brain MRI did not show definite evidence of distant metastatic disease.

The patient received neoadjuvant chemotherapy with doxorubicin and cyclophosphamide every 2 weeks for 4 cycles followed by weekly paclitaxel for 12 weeks. Followup CT of the chest, abdomen, and pelvis after neoadjuvant chemotherapy showed a partial response with no evidence of distant metastases. The patient subsequently underwent right modified radical mastectomy with axillary lymph node dissection. Pathology results showed multifocal residual disease measuring up to $16.5 \mathrm{~cm}$, with $>10$ additional satellite tumors and skin involvement. Among resected axillary lymph nodes, 7 of 9 sampled lymph nodes were positive for metastatic disease, with the largest deposit measuring $4.5 \mathrm{~cm}$ with extracapsular extension. After surgery, the patient received adjuvant radiation with 5,040 cGy to the right chest wall, 6,040 cGy to the right chest wall scar, and $4,500 \mathrm{cGy}$ to the right regional lymphatics. After completion of radiation, she began adjuvant endocrine therapy using letrozole.

Approximately 10 months later, the patient presented again with skin nodules on the right chest wall while on letrozole. Subsequently, a punch skin biopsy was performed that showed recurrent infiltrating ductal carcinoma. At that time, a PET/CT scan showed hypermetabolic activity in the right chest wall mass with no 
definite evidence of distant metastases. The patient then underwent resection of the right chest wall lesion. Pathology results showed recurrent invasive adenocarcinoma consistent with breast primary, and ER-positive by IHC at $76 \%$ to $100 \%$, PR-negative $<1 \%$, and no HER2 overexpression (IHC 0). Further quantification of stromal tumor-infiltrating lymphocytes (sTILs) based on the International TILs Working Group ${ }^{1}$ showed $<2 \%$ sTILs (Figure 1). The PD-L1 IHC 22C3 pharmDx IHC assay (Dako; Carpinteria, CA) showed PD-L1 expression $<1 \%$ in both tumor and immune cells (Figure 2). The chest wall lesion was further evaluated for potentially actionable genomic alterations using next-generation sequencingbased comprehensive genomic profiling (CGP) with the FoundationOne test. CGP showed high tumor mutational burden (TMB) (40 mutations/megabase [mut/Mb]) and likely pathogenic alterations in PIK3CA (E453Q, E545K), ESR1 (E380Q), MEN1 (S313*), CTCF (E145*), GATA3 (M294K), MAP3K1 (S782*), MLL3 (S2025*), NUP93 (Q15*), SPEN (E376*), SPTA1 (Q2195*), and TP53 (R248Q) and ZNF703 amplification, but no evidence of mismatch repair deficiency (dMMR). At that time, her systemic treatment was switched to fulvestrant and palbociclib.

Approximately 3 months later, the patient's followup PET/CT scan unfortunately showed progressive disease with contralateral left axillary lymphadenopathy, which was confirmed by fine-needle aspiration biopsy showing metastatic disease consistent with breast primary, ER $>90 \%, \mathrm{PR}>50 \%$, and no HER2 overexpression (IHC 0). Given that this was the only site of recurrence or progressive disease, the patient underwent excision of these lymph nodes. Pathology results showed that 1 of 7 left axillary lymph nodes was positive for metastatic

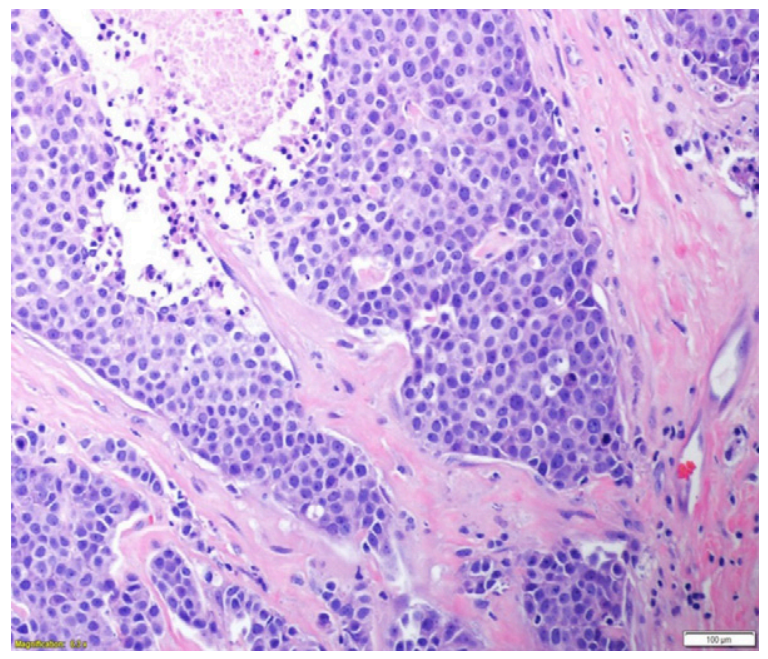

Figure 1. Chest wall mass consistent with adenocarcinoma. Hematoxylin-eosin stain showing $<2 \%$ stromal tumor-infiltrating lymphocytes (original magnification, $\times 100$ ). moderately differentiated adenocarcinoma consistent with breast primary, measuring $3.0 \mathrm{~cm}$ with extranodal extension. She was continued on fulvestrant and palbociclib after surgery. The patient had another follow-up PET/CT scan 3 months later that unfortunately showed progressive disease with 2 new soft tissue lesions in the right fourth and fifth intercostal space and a soft tissue nodule in the right fourth costochondral junction. Her treatment was then switched to weekly dosing of capecitabine at $1,500 \mathrm{mg}$ orally twice daily, 7 days on and 7 days off. Because of high TMB seen on the FoundationOne testing, off-label use of nivolumab was requested through the patient access program with Bristol-Myers Squibb and was added 43 days later after the patient was started on capecitabine. At the time of the last follow-up, the patient remained on both capecitabine and nivolumab. She had achieved complete response with no evidence of disease lasting $>26$ months (Figure 3). The patient's informed consent was obtained for this case report.

In collaboration with Foundation Medicine, we further evaluated hybrid capture-based CGP of up to 395 cancer-related genes using the FoundationOne assay in 14,867 histologically defined breast carcinomas sequenced at Foundation Medicine. Molecular subtype was available for a subset of patients. ER status was available in 2,394 patients, of whom $1,371(57 \%)$ had ER-positive (ER+) disease. HER2 status was available in 14,867 patients, of whom 1,522 (10\%) had HER2-positive (HER2+) disease. Among 2,394 patients with available ER and HER2 status, 917 (38\%) had ER-negative/HER2negative breast cancer. There were 5,241 samples from locoregional lesions and 6,710 samples from metastatic lesions. PD-L1 expression status using the VENTANA

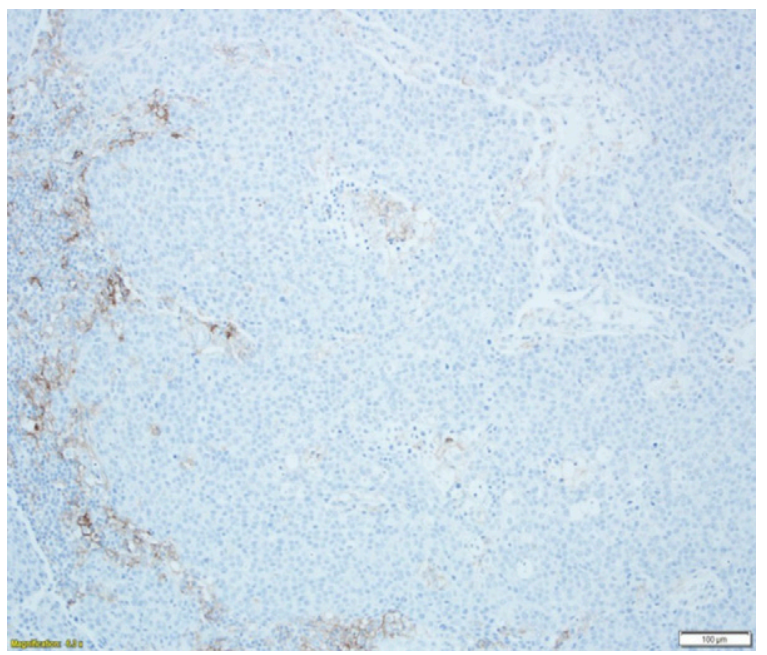

Figure 2. Biopsy of the chest wall lesion showing negative PD-L1 expression with $22 \mathrm{C} 3$ pharmDx IHC assay (original magnification, $\times 40$ ). 

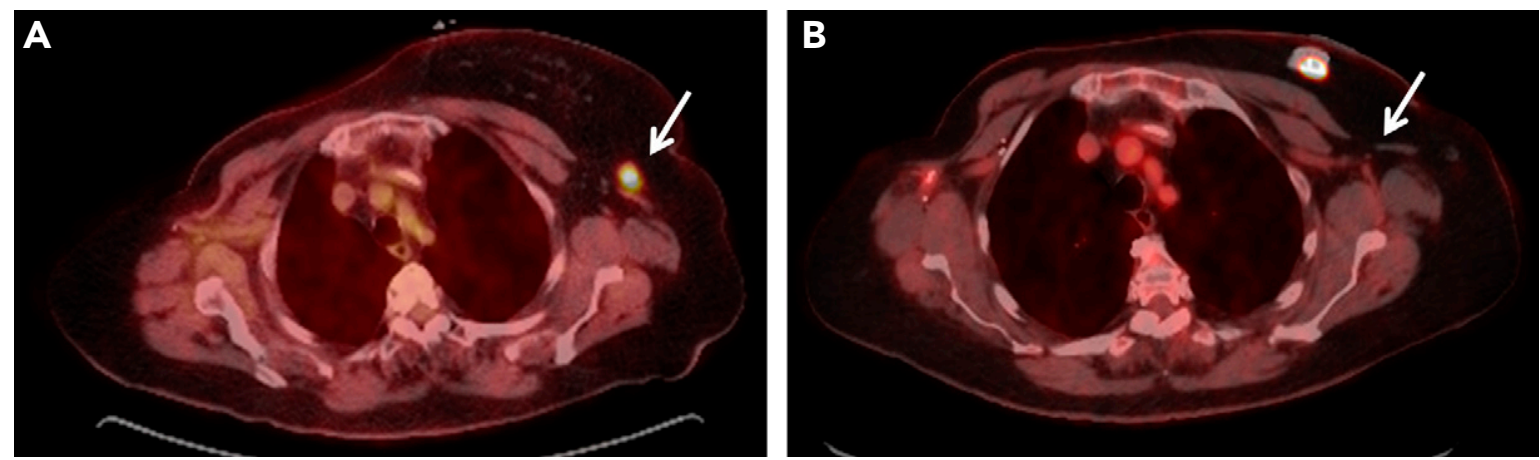

Figure 3. PET/CT from (A) August 2017 showing hypermetabolic lesion in the left axilla (white arrow), and (B) August 2019 showing complete resolution of hypermetabolic lesions in the left axilla (white arrow).

PD-L1 assay with the SP142 antibody (F. Hoffmann-La Roche Ltd) was available in 1,425 samples. We defined PD-L1 positivity as $\geq 1 \%$ tumor staining. We found that PD-L1 expression data were available in 99 triplenegative breast cancer (TNBC) samples, 134 HER2+ breast cancer samples, and $129 \mathrm{ER}+$ breast cancer samples. High TMB was defined as $\geq 10$ mut/Mb.

Consistent with previous reports, ${ }^{2}$ the incidence of PD-L1 positivity was highest in TNBC at $14 \%$, followed by $6.0 \%$ in HER 2 - disease and $5.1 \%$ in invasive lobular carcinoma, and lowest in ER+ disease at $2.3 \%$. Interestingly, the percentage of PD-L1-positive cases was significantly lower in metastatic disease compared with local disease ( $6.3 \%$ vs $11.1 \% ; P=.005)$. The frequency of high TMB was highest in invasive lobular carcinoma at $13.6 \%$, followed by HER2 + disease at $9.9 \%$, TNBC at $7.0 \%$, and ER+ disease at $6.9 \%$. High TMB was associated with older age, with $3.7 \%$ in patients aged $\leq 45$ years, $9.3 \%$ in patients aged 46 to 60 years, and $12.8 \%$ in patients aged $\geq 61$ years $(P<.001)$. Using the cutoff of $\geq 10 \mathrm{mut} / \mathrm{Mb}$, PD-L1 positivity and TMB-high populations were not significantly overlapping (odds ratio, 1.02; $P=.87$ ). High TMB was also associated with metastatic disease, with $11.1 \%$ of metastatic lesions harboring high TMB compared with $5.3 \%$ with localized disease $(P<.001)$. Among patients with very high $\mathrm{TMB}$, defined as $>20 \mathrm{mut} / \mathrm{Mb}$, there was a significant association between high TMB and PD-L1 positivity (odds ratio, 2.6; $P=.023$ ). Nevertheless, even with a high cutoff of $>20$ mut/Mb, $79 \%$ of the TMB-high samples were PD-L1-negative.

There are several mechanisms underlying genomic instability in breast cancer, including homologous recombination deficiency, dMMR, and apolipoprotein B mRNA editing enzyme, catalytic polypeptide-like (APOBEC) cytidine deaminase. ${ }^{3}$ Emerging data have shown that dysregulation of APOBEC enzyme can result in tumors with high TMB. ${ }^{4}$ Further evaluation of this particular patient's tumor genomic landscape showed no evidence of dMMR or homologous recombination repair deficiency. Using the trinucleotide mutational signatures described by Zehir et al, ${ }^{5}$ we found that this patient's tumor harbored a dominant APOBEC trinucleotide signature, with a fit of $91 \%$. In the FoundationOne cohort, most of the TMB-high ( $\geq 10$ mut/Mb) breast samples with at least 10 assessable mutations were APOBEC mutational signature-positive (62.3\%; 909 of 1,460). Across molecular subtypes, the fraction of TMB-high cases with an APOBEC signature was relatively similar, including $56.3 \%$ ( 27 of 48 ) in TNBC, $69.2 \%$ (54 of 78) in ER+ disease, and $62.4 \%$ (111 of 178) in HER2 + disease. Comparing between ductal and lobular carcinoma, we noted that TMB-high lobular carcinomas exhibited a higher proportion of APOBEC positivity $(85.8 \%$; 121 of 141$)$ relative to TMB-high ductal carcinomas (56.0\%; 200 of 357; $P<.0001)$. In addition, TMB-high samples from metastatic sites harbored a significantly higher fraction of APOBEC positivity relative to TMB-high samples from primary tumors $(67.3 \%$ vs $52.3 \%$; $P<.0001)$. Approval for this particular part of the study, including a waiver of informed consent and a HIPAA waiver of authorization, was obtained from the Western Institutional Review Board (protocol number 20152817).

\section{Discussion}

Immunotherapy using immune checkpoint inhibitors (ICIs) has truly revolutionized cancer therapy in the past decade. ICIs exert their activity mainly through enhancing preexisting host immune response. Multiple clinical trials have shown long durable responses using ICIs, particularly in immunogenic or "hot" tumors such as melanoma or lung cancer. In contrast, results of single-agent ICIs in less-immunogenic tumors, including breast cancer, have been less promising, particularly in immunologically "cold" tumors such as hormone receptorpositive, HER2-negative, or luminal breast cancer.

One way to evaluate preexisting host immune response is by measuring TILs. A higher percentage of TILs has been shown to be associated with improved outcomes in patients treated with ICIs in multiple tumor types. ${ }^{6}$ 
In breast cancer, several studies have also shown the prognostic value of TILs beyond other conventional pathologic characteristics and staging. ${ }^{7-9}$ An initial study showed that each $10 \%$ increase in TILs was asso ciated with a $15 \%$ to $17 \%$ reduction in risk of relapse in patients with TNBC. ${ }^{7}$ More recently, 2 additional pooled individual analyses from multiple large clinical trials confirmed the prognostic significance of TILs in patients with early-stage breast cancer, particularly in TNBC. ${ }^{8,9}$ Nevertheless, similar to our case report, a number of studies have shown a lower preexisting host immune response measured by TILs in cold luminal breast cancer. ${ }^{8,10}$

Several clinical trials have evaluated the efficacy of ICIs in breast cancer. ${ }^{11}$ For example, TNBC is a subtype for which ICIs seem to have promising activity. The objective response rates (ORRs) of single-agent ICIs were reported to be as high as $23.1 \%$ in patients with TNBC. ${ }^{12}$ In contrast, the previous phase IB JAVELIN trial showed that the ORR of the single-agent anti-PD-L1 avelumab was only $2.8 \%$ in patients with unselected luminal breast cancer. ${ }^{13}$

Currently, there are several potential biomarkers to identify patients who are more likely to benefit from ICIs. In addition to baseline TILs, PD-L1 expression and microsatellite instability status or dMMR have been shown to correlate with an improved outcome using ICIs. ${ }^{6}$ Impairment of MMR can result in genomic instability and hypermutated tumors with highly immunogenic frameshift peptide neoantigens, which can trigger immune response and increase T-cell activation. ${ }^{14}$ In 2017, the FDA approved pembrolizumab for the treatment of patients with solid tumors with dMMR regardless of their tumor type, based on the phase II trial by Le et al, ${ }^{15}$ which reported an ORR of $40 \%$ in patients with dMMR colorectal cancer and $71 \%$ in those with dMMR noncolorectal cancer. In a subsequent report, ORRs were observed in $53 \%$ of patients with dMMR across 12 tumor types, with $21 \%$ being complete responses. ${ }^{16}$ However, the incidence of dMMR in breast cancer has been reported to be fairly low at $1.53 \%$ compared with other cancers, such as $31.37 \%$ in endometrial cancer and $19.72 \%$ in colon cancer. ${ }^{17}$

In addition to dMMR status, recent studies have shown that TMB itself is also predictive of improved outcome in patients receiving ICIs. Initial studies in melanoma have shown that high TMB was associated with improved outcomes in patients receiving antiCTLA-4 antibodies. ${ }^{18,19}$ Similar results were also observed in non-small cell lung cancer, ${ }^{20}$ melanoma, ${ }^{21}$ and urothelial cancer ${ }^{22}$ treated using anti-PD-1/PD-L1 therapies. More recently, Samstein et $\mathrm{al}^{23}$ examined the predictive value of TMB across multiple tumor types, with 1,662 advanced cancers treated using ICIs. Overall, higher TMB was associated with improved survival in patients receiving ICIs in most tumor types. However, TMB cutoffs seemed to be markedly varied between different cancer types. ${ }^{24}$

Our unique case report highlights the benefit of nextgeneration sequencing beyond standard histopathologic features. Although this patient's tumor is considered to be immunologically cold by conventional clinicopathologic factors, including luminal breast cancer histology, low sTILs, and PD-L1 negativity, the patient did derive prolonged durable benefit from ICIs. Further evaluation in the larger breast cancer cohort showed that TMBhigh PD-L1-positive populations were not significantly overlapping. Unexpectedly, up to $79 \%$ of TMB-high tumors were PD-L1-negative. Similar findings were reported previously by Barrett et $\mathrm{a}^{25}$ in a smaller group of patients with TNBC, wherein PD-L1 expression by IHC did not seem to correlate with TMB, dMMR status, and amount of predicted neoepitopes. Nonetheless, profound response to ICIs in patients with TNBC with PDL1-negative tumors harboring dMMR has been reported. ${ }^{26}$ Similar observations were also previously reported by Rizvi et $\mathrm{al}^{20}$ in non-small cell lung cancer. Overall, patients with both TMB-high and PD-L1-positive tumors had the highest durable clinical benefit rate of $91 \%$. Interestingly, among patients with low PD-L1 expression, $75 \%$ of those with TMB-high tumors had durable clinical benefit compared with $11 \%$ with TMB-low tumors. Based on these intriguing data, additional studies will be needed to evaluate the efficacy of ICIs in this group of patients with breast cancer with high TMB but PD-L1-negative tumors.

Notably, given that our patient also received capecitabine, it is unclear whether her disease would have responded to single-agent capecitabine or single-agent ICI. Nevertheless, because her disease progressed rather rapidly with multiple lines of previous therapies, it is unlikely that she would have benefited from single-agent capecitabine alone. Because several recent studies showed the benefit of combining chemotherapy with immunotherapy, it is conceivable that our patient derived the most benefit from the combination of capecitabine and nivolumab. For treatment of TNBC, the FDA recently approved combination nab-paclitaxel and atezolizumab for patients with unresectable locally advanced or metastatic TNBC, based on the report from the phase III IMpassion 130 trial. ${ }^{27}$ Nevertheless, this particular combination seemed to only benefit patients with PD-L1-positive tumors. In luminal breast cancer, similar results using the combination of ICI and chemotherapy were also reported in the I-SPY 2 trial (ClinicalTrials.gov identifier: NCT01042379). ${ }^{28}$ In this trial, the addition of pembrolizumab to the standard neoadjuvant chemotherapy resulted in a higher complete pathologic response rate of $34 \%$ compared with $13 \%$ using standard neoadjuvant chemotherapy alone. 


\section{Conclusions}

This case report highlights that tumors with high TMB may still respond to ICIs in combination with chemotherapy, despite patients having tumors that may have been considered immunologically cold based on standard clinicopathologic assessment. Although the tumor in this case was PD-L1-negative and had no evidence of dMMR, the patient's disease exhibited a durable complete response to the combination of nivolumab and capecitabine for a prolonged period. Further evaluation with CGP in a large breast cancer cohort showed that PD-L1 positivity and TMB-high populations were only moderately overlapping. A considerable number of breast tumors with high TMB were PD-L1-negative. Future studies will be needed to further evaluate the efficacy of ICIs in patients with breast cancers with high TMB and/or an APOBEC trinucleotide signature that are considered immunologically cold based on other conventional pathologic assessments.

Submitted September 10, 2019; accepted for publication January 30, 2020.

Author contributions: Obtained FoundationOne data: Sokol. Obtained pathology images: Nassar. Manuscript preparation: Chumsri. Manuscript editing: Sokol, Soyano-Muller, Parrondo, Reynolds, Thompson. Final approval of manuscript: Sokol, Soyano-Muller, Parrondo, Reynolds, Thompson.

Disclosures: Dr. Sokol has disclosed that he is employed by Foundation Medicine and that he owns stock in Roche. The remaining authors have disclosed that they have not received any financial consideration from any person or organization to support the preparation, analysis, results, or discussion of this article.

Funding: Research reported in this publication was supported by the Breast Cancer Research Foundation (BCRF-17-161).

Correspondence: Saranya Chumsri, MD, Robert and Monica Jacoby Center for Breast Health, Mayo Clinic, 4500 San Pablo Road South, Jacksonville, FL 32224. Email: chumsri.saranya@mayo.edu

\section{References}

1. Salgado R, Denkert C, Demaria S, et al. The evaluation of tumor-infiltrating lymphocytes (TILs) in breast cancer: recommendations by an International TILs Working Group 2014. Ann Oncol 2015;26:259-271.

2. Zhang $M$, Sun $H$, Zhao S, et al. Expression of PD-L1 and prognosis in breast cancer: a meta-analysis. Oncotarget 2017;8:31347-31354.

3. Nik-Zainal S, Morganella S. Mutational signatures in breast cancer: the problem at the DNA level. Clin Cancer Res 2017;23:2617-2629.

4. Wang $\mathrm{S}$, Jia M, He Z, et al. APOBEC3B and APOBEC mutational signature as potential predictive markers for immunotherapy response in non-small cell lung cancer. Oncogene 2018;37:3924-3936.

5. Zehir A, Benayed R, Shah RH, et al. Mutational landscape of metastatic cancer revealed from prospective clinical sequencing of 10,000 patients. Nat Med 2017:23:703-713.

6. Nishino M, Ramaiya NH, Hatabu H, et al. Monitoring immune-checkpoint blockade: response evaluation and biomarker development. Nat Rev Clin Oncol 2017;14:655-668.

7. Loi $S$, Sirtaine N, Piette F, et al. Prognostic and predictive value of tumorinfiltrating lymphocytes in a phase III randomized adjuvant breast cancer trial in node-positive breast cancer comparing the addition of docetaxel to doxorubicin with doxorubicin-based chemotherapy: BIG 02-98. J Clin Oncol 2013;31:860-867.

8. Denkert C, von Minckwitz G, Darb-Esfahani S, et al. Tumour-infiltrating lymphocytes and prognosis in different subtypes of breast cancer: a pooled analysis of 3771 patients treated with neoadjuvant therapy. Lancet Oncol 2018;19:40-50.

9. Loi S, Drubay D, Adams S, et al. Tumor-infiltrating lymphocytes and prognosis: a pooled individual patient analysis of early-stage triplenegative breast cancers. J Clin Oncol 2019;37:559-569.

10. Miyoshi $Y$, Shien T, Ogiya A, et al. Associations in tumor infiltrating lymphocytes between clinicopathological factors and clinical outcomes in estrogen receptor-positive/human epidermal growth factor receptor type 2 negative breast cancer. Oncol Lett 2019;17:2177-2186.

11. Solinas C, Gombos A, Latifyan $\mathrm{S}$, et al. Targeting immune checkpoints in breast cancer: an update of early results. ESMO Open 2017;2:e000255.

12. Nanda R, Chow LQ, Dees EC, et al. Pembrolizumab in patients with advanced triple-negative breast cancer: phase Ib KEYNOTE-012 study. J Clin Oncol 2016;34:2460-2467.

13. Dirix LY, Takacs I, Jerusalem G, et al. Avelumab, an anti-PD-L1 antibody, in patients with locally advanced or metastatic breast cancer: a phase $1 \mathrm{~b}$ JAVELIN solid tumor study. Breast Cancer Res Treat 2018;167:671-686.
14. Kloor M, von Knebel Doeberitz M. The immune biology of microsatelliteunstable cancer. Trends Cancer 2016;2:121-133.

15. Le DT, Uram JN, Wang H, et al. PD-1 blockade in tumors with mismatchrepair deficiency. N Engl J Med 2015;372:2509-2520.

16. Le DT, Durham JN, Smith KN, et al. Mismatch repair deficiency predicts response of solid tumors to PD-1 blockade. Science 2017;357:409-413.

17. Bonneville R, Krook MA, Kautto EA, et al. Landscape of microsatellite instability across 39 cancer types [published online October 3, 2017]. JCO Precis Oncol, doi: 10.1200/PO.17.00073

18. Snyder A, Makarov V, Merghoub T, et al. Genetic basis for clinical response to CTLA-4 blockade in melanoma. N Engl J Med 2014;371:2189-2199.

19. Van Allen EM, Miao D, Schilling B, et al. Genomic correlates of response to CTLA-4 blockade in metastatic melanoma. Science 2015;350:207-211.

20. Rizvi NA, Hellmann MD, Snyder A, et al. Cancer immunology. Mutational landscape determines sensitivity to PD-1 blockade in non-small cell lung cancer. Science 2015;348:124-128.

21. Hugo W, Zaretsky JM, Sun L, et al. Genomic and transcriptomic features of response to anti-PD-1 therapy in metastatic melanoma. Cell 2016;165:35-44.

22. Rosenberg JE, Hoffman-Censits J, Powles T, et al. Atezolizumab in patients with locally advanced and metastatic urothelial carcinoma who have progressed following treatment with platinum-based chemotherapy: a single-arm, multicentre, phase 2 trial. Lancet 2016;387:1909-1920.

23. Samstein RM, Lee $\mathrm{CH}$, Shoushtari $\mathrm{AN}$, et al. Tumor mutational load predicts survival after immunotherapy across multiple cancer types. Nat Genet 2019;51:202-206.

24. Galuppini F, Dal Pozzo CA, Deckert J, et al. Tumor mutation burden: from comprehensive mutational screening to the clinic. Cancer Cell Int 2019;19:209.

25. Barrett MT, Lenkiewicz E, Malasi S, et al. The association of genomic lesions and PD-1/PD-L1 expression in resected triple-negative breast cancers. Breast Cancer Res 2018;20:71.

26. Kok M, Horlings HM, Snaebjornsson P, et al. Profound immunotherapy response in mismatch repair-deficient breast cancer [published online October 3, 2017]. JCO Precis Oncol, doi: 10.1200/PO.17.00052

27. Schmid P, Adams S, Rugo HS, et al. Atezolizumab and nab-paclitaxel in advanced triple-negative breast cancer. N Engl J Med 2018;379: 2108-2121

28. Nanda R, Liu MC, Yau C, et al. Pembrolizumab plus standard neoadjuvant therapy for high-risk breast cancer (BC): results from I-SPY 2 [abstract]. J Clin Oncol 2017;35(Suppl):Abstract 506 